\title{
DESIGN, FABRICATION AND TESTING OF A BOUNDED WAVE EMP SIMULATOR FOR MIL - STD - 461 E/F
}

\author{
Shubha Parochi ${ }^{1}$, Kanchan Cecil ${ }^{2}$ \\ ${ }^{l}$ Jabalpur Engineering College, Jabalpur) \\ $\left({ }^{2}\right.$ Asst.Prof. , Jabalpur Engineering College, Jabalpur)
}

\begin{abstract}
A parallel-plate test cell is designed and implemented for EMP testing covered under MIL STD 461E/F, testing standard RS-105, while being two-man portable, and set-up in less than one hour. The MIL STD 461 E/F document details the RS-105 hardware requirements for this test. This includes a TEM waveguide structure, a pulse generator and a Faraday cage for shielding the oscilloscope used for calibrating and diagnosing the electrical behavior of the system. The specification suggests a solid parallel plate TEM waveguide, sourced by a pulse generator. A field probe is to be used to measure the field strength at various defined points within the test region. Design and operational data from the tester are presented in this paper.
\end{abstract}

Keywords: - Antenna, Bounded-wave, Electromagnetic interference (EMI), Electromagnetic pulse (EMP), Fast rise time.

\section{I.INTRODUCTION}

The bounded wave electromagnetic pulse (EMP) simulator is used to simulate the EMP which is generated by nuclear weapons at a high altitude (HEMP). The world started to develop EMP simulator in the 1960s. Now many countries already have many EMP simulators of different models. The simulator provides the simulated high electromagnetic pulse (HEMP) environment for testing smaller objects such as cable and other electrical equipment in vehicles, under the condition of low source power. The property of small bounded wave EMP simulator satisfies demand of relational standards. It is enough for testing smaller objects. A test apparatu s is desired to test electronic assemblies and enclosures under MIL STD $461 \mathrm{E} / \mathrm{F}, \mathrm{RS}-105$ test procedure, to test for electronic vulnerability to an Electro Magnetic Pulse (EMP). Nowadays, MIL-STD-461E which was published in 1999 is referred widely. In the standard, it is defined that the model of EMP is a double exponential pulse and it has a peak amplitude of $50 \mathrm{kV} / \mathrm{m}$ shown in the figure 1.1 , a rise time in the range of $1.8 \mathrm{~ns}-2.8 \mathrm{~ns}$, a pulse width at half maximum

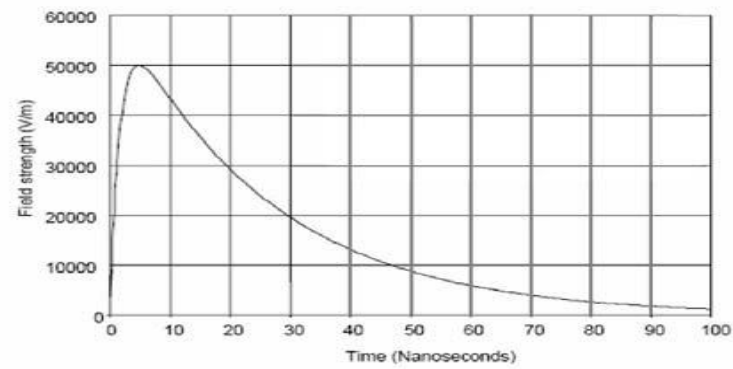

Fig 1.1 Electro Magnetic Pulse

$23 \mathrm{~ns} \pm 5 \mathrm{~ns}$ [1].The maximum test volume of EUT is $20 \mathrm{~cm} \mathrm{X} 50 \mathrm{~cm} \mathrm{X} 12 \mathrm{~cm}$ (L X WX H). It is enough for the testing of smaller objects. [1], with a system that is very simple to operate and is two-man portable for field testing.

\section{II.INITIAL DESIGN AND BUILDING OF SMALL SIMULATOR}

The small EMP simulator comprises a parallel plate (working volume) and two sloping triangular plates joined to the parallel plates. One end is driven by a pulser, and the other end is terminated by a resistor. The geometry of small EMP simulator makes sure that the characteristic impedance is constant. In order to make the wave, in the working volume, close to plane wave is must to be ensured that [4]

- The width of the waveguide should be twice the width of the EUT,

- The length of the test region should be twice the length of the EUT.

- The height or the plate separation should be 3 times the height of the EUT . 
- It is must to be ensured that 1 (the length of the first transition section) is twice more than $\mathrm{h}$ (the height of the simulator).

- The dimensions of simulator are chosen to provide equal characteristic impedance.

For design of the transition section The input impedance of TEM horns is dependent both on the flare angle $\varnothing$ and the apex angle of the plates, $\Phi$.the input impedance of antenna is generally required to match that of the source voltage.[4]

We require apex angle of $30 \mathrm{deg}$, which gives width of $115.4 \mathrm{~cm}, \tan 30^{\circ}=\mathrm{W} / \mathrm{T} . \mathrm{L}$,

where $\mathrm{W}=$ Width and $\mathrm{T} . \mathrm{L}=$ Transitional length

and flare angle of $21.5 \mathrm{deg}$, which gives height of $36.7 \mathrm{~cm}$, sin $21.5^{\circ}=36.7 \mathrm{~cm}$.

The impedance $\mathbf{Z}_{0}$ of an isolated transmission line is given by

$\mathrm{Z}_{0}=\delta \mathrm{a} / \mathrm{b}\{1+\mathrm{a} / \mathrm{b} \pi[1+\ln 2 \pi / \mathrm{a} / \mathrm{b}]\}^{-1}$

$\delta=377 \Omega$, a = plate separation, and $\mathrm{b}$ - plate width.

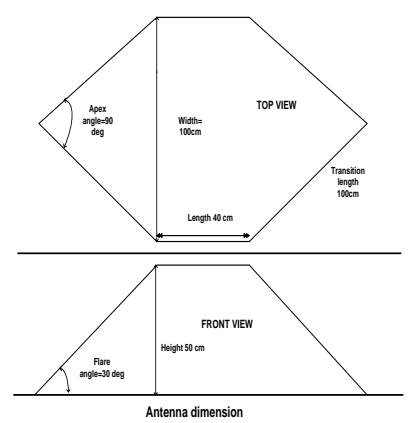

Fig 2.1 Top and Front View of antenna

\section{III.GENERATOR}

The generator consists of a high voltage power supply (HVPS), a capacitor and a spark gap. The capacitor can be charged by a commercial available HVPS The capacitor is discharged via the spark gap and the transmission line into the load resistor. The capacitor and spark gap can be housed in a coaxial structure that can be connected to the TEM cell and matched to the characteristic impedance of the line. In this way the generator can easily be disconnected from the TEM cell. A low inductance, high voltage capacitor is needed. Many factors must be considered in order to make a proper choice. In our case, properties should include: peak voltage, peak current, wave shape, duty cycle, dissipation factor, power factor, life expectancy, temperature coefficient, etc. Other important characteristics are: the equivalent series resistance, impedance, self-resonant frequency, voltage reversal. In general, ceramic capacitors have small dimensions, low inductance, low impedance and can handle high voltages. On the other hand they are temperature and voltage dependent. If the temperature of the room is stable and the repetition rate is not too high, ceramic disc capacitors may be a suitable choice [3]

\subsection{DESIGN OF GENERATOR SWITCH}

It can be built along the principle of the discharge of a previously charged $120-\Omega$ pulse-forming line by means of a circuit breaker (here, a high-pressure gas switch) in an impedance circuit equal to its characteristic impedance. The basic factors involved in designing spark gaps are the field geometry, gap length, dielectric gas filling and its pressure, load characteristics, repetition rate requirements, and temporal history of the applied voltage. The switch is made of two brass electrodes with tungsten hemispheres (diameter $=10 \mathrm{~mm}$ ) at their end. Tungsten, graphite, or elkonite limit electrode erosion to a minimum. The rise time of the spark gap is dependent on the inductance of the spark gap and on the resistive phase of the spark.[11]

$\mathrm{T}_{\mathbf{R}}\left(\right.$ risetime ) ns $=\frac{88(\rho)^{0.5}}{\left({ }_{\mathrm{L}}^{\mathrm{Z}}\right)^{1 / 3 *(E)^{4 / 3}}\left(\rho_{0}\right)}$

Where $\mathrm{Z}_{\mathrm{L}=120 \Omega} \Omega$ load impedance, $\rho_{0}=$ air density,$\rho=\mathrm{N}_{2}$ density, where $\mathrm{E}$ is the field strength in $\mathrm{KV} / \mathrm{mm}$ and (p/po) is the ratio of the gas density to that of air at STP (standard temperature and pressure).[11] The highpressure gas switch technology is widely used because the dielectric strength of gas allows adequate voltage levels and because the gas quickly regains its dielectric strength after the discharge. So, this technology can be designed for sub nanosecond time scales, and the stray inductance generator has to be reduced sufficiently to generate sub nanosecond rise times.[14] The DC breakdown strength of a spark gap with nitrogen in a uniform field is given as: 
Voltages in KV, Pressure in Bar, Distance in mm.To decrease the inductance of the spark gap the dimensions

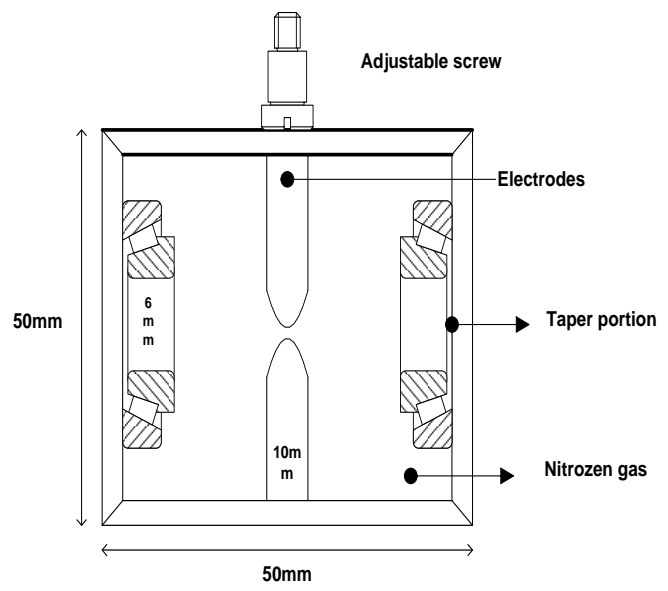

Switch design

should be made as small as possible.

\section{IV.SYSTEM SPECIFICATIONS}

- Transmission line- parallel plates $115.4 \mathrm{~cm}$ wide, $40 \mathrm{~cm}$ long, $36.7 \mathrm{~cm}$ separation, $120 \Omega$ impedance.

- Power supply - $22 \mathrm{v}-30 \mathrm{kV}$ dc to dc supplies.

- energy storage capacitors $-220 \mathrm{pf}$

Capacitors.

- $\quad$ spark gaps - pressurized switch

- pulse shape - exponential

- rise time - 1.8-2.5 nsec (10-90 percent)

- fall time - $18 \mathrm{nsec}$

- peak field strength - $10-30 \mathrm{kV} / \mathrm{m}$

- $\quad$ system line impedance - $120 \Omega$.

\section{V.THE PROPAGATION OF EMP AND ITS EFFECT OF RISE TIME}

The rise time of pulser is $2 \mathrm{~ns}$. However, the rise time of EMP in the test volume has delayed to $2.3 \mathrm{~ns}$. That is the result of the coordinate action of the rise time (simulator) and the reflected wave. The relation of the rise time of simulator and the length of the first transition section is given by equation [1].

$T_{d}=\left\{\left(l^{2}+h^{2}\right)^{0.5}-1\right\} /$

$\mathbf{T}_{\mathrm{d}}=339 \mathrm{ps}$

Where, 1 is the length of the first transition, $\mathrm{h}$ is the height of test volume; $\mathrm{c}$ is the propagating rate of electromagnetic wave in the air. It is known from equation (4) that the rise time of simulator is $0.339 \mathrm{~ns}$. So we can know that the effect of reflected wave is more important. [1]

\subsection{REFLECTIONS}

An abrupt change at the junctions between taper and line will introduce a capacitive mis-match and result in the mentioned reflections. A radius introduced at these points will minimize this effect and improve the design. The reflection at the junction of the parallel-plate section and the termination (output) taper can be reduced in the same way as with the input taper. A smaller length of the output taper results in larger reflections and shorter clear times of the multiple reflections [2].

\section{VI.SYSTEM TESTING AND DIAGNOSIS RESULTS}

A sample test electric field waveform measured inside the test volume The waveform suggests a number of problems with the line. The initial portion of the pulse is characterized by excessive ringing. Approximately 20 ns into the pulse, reflections from the load begin to appear, and approximately 60 seconds into the pulse, more load reflections are observed. The multiple reflections from the load are problematic since they can force the system to fall out of specification. As the high voltage pulse is launched onto the TEM structure, two waves are generated in section D: a free wave that is generated as a consequence of the input appearing much like a linear 
half TEM horn antenna $\mathbf{1}$, and a bounded wave that propagates the line $\mathbf{2}$. The bounded wave $\mathbf{1}$ continues through the transmission line and is mostly terminated by the load resistor. Reflected energy $\mathbf{3}$ from the load, due to impedance mismatches between the line and the load, propagates back into the test volume. The free wave 2 continues toward the load, although non uniform as the wave is aimed slightly off the horizontal. Some of the free wave energy reflects off the solid load-side waveguide and returns to the test volume $\mathbf{5}$.

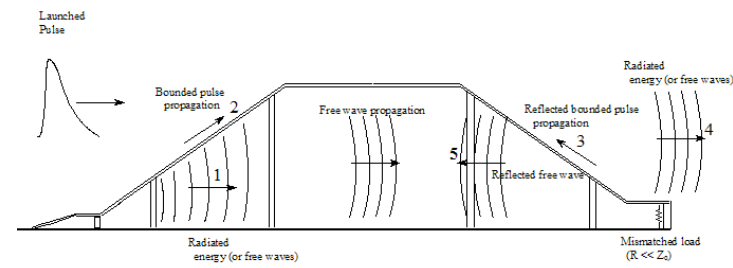

D

$\mathrm{F}$

Fig 6.1 Illustration of the Waves Propagating in the TEM Structure.

Simulations were made and the results of Fig 6.2 indicate the launching of a pulse down the antenna structure, the current remains uniform on each of the rods, instead of migrating toward the edges. Furthermore, the electric field in the test region is much more homogeneous.

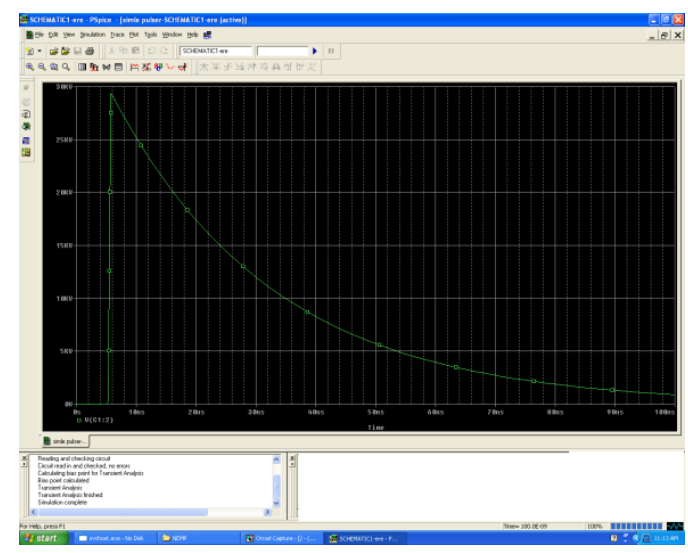

Fig 6.2 Simulation Results for generating EMP Pulse inside simulator.
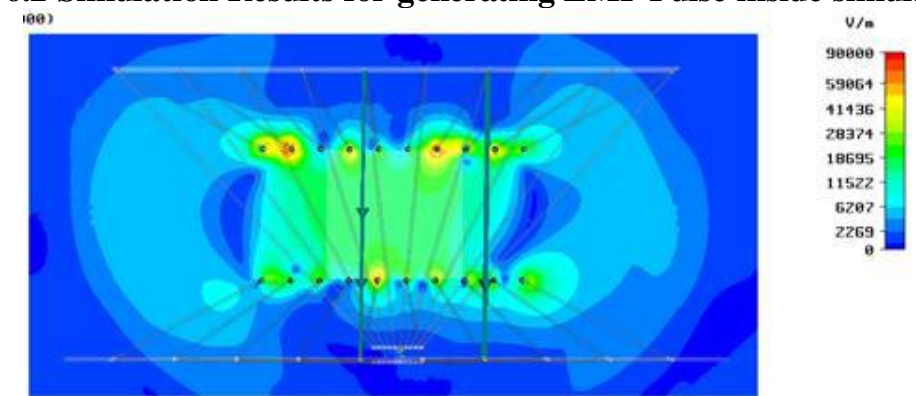

Fig 6.3Simulation Results Electric Field Pattern Inside the Test Volume .

\section{CONCLUSION}

A small EMP simulator with a low power pulser is designed in this paper. We get three conclusions by analyzing its electromagnetic property as follow.

(1) The property of double exponential is quite retained by the EMP in the test volume. It has a peak field of $30 \mathrm{kV} / \mathrm{m}$, a rise time of $2.8 \mathrm{~ns}$, a pulse width at half maximum of $18 \mathrm{~ns}$. The simulated HEMP environment has been produced in the test volume of small EMP simulator.

(2) The distribution of electronic field in frequency domain is almost the same. The test volume measured by the distribution in time domain is $20 \mathrm{~cm} \mathrm{X} 50 \mathrm{~cm} \mathrm{X} 12 \mathrm{~cm}(\mathrm{~L} \mathrm{X} \mathrm{W} \mathrm{X} \mathrm{H)}$. That is enough for the small test objects such as some electronically equipment in vehicles. 

$461 \mathrm{E} / \mathrm{F}$

(3) The low frequency component of the reflected wave which is produced at junction occupies more power, So the rise time of EMP is delayed. It is still a problem to resolve in the future.

\section{VIII .REFERENCES}

1. The Design and Property Analysis of Small Wave Bounded EMP Simulator, linliang Cai, Bo Wang', Fengye Hu, Delong Zeng School of Communication Engineering, Jilin University ,Changchun, China ,chinawang200 I @ sina.com*, Gang Guo ,Department of Control Engineering, Aviation University of Air-Force Changchun, China. 2010 International Conference on Computer, Mechatronics, Control and Electronic Engineering (CMCE).

2. DESIGN OF A BOUNDED WAVE EMP SIMULATOR (Intended as second stage simulator for DREO) by P.A.A. Sevat Nuclear Effects Ser:tion ,ceCes, o'. Electronics Division.

3. DESIGN OF A TEM CELL EMP SIMULATOR (U) by Pete Sevat Nuclear Effects Section Electronics Division, DEFENCE RESEARCH ESTABLISHMENT OTTAWA REPORT NO. 1084 PCN June 1991041 LT Ottawa.

4. Input Inpedance calcution for TEM Horn Antennas, BY Geoff Staines and Sean Braidwood,Electronic Warfare division Electronics and Surveillance Research Laboratory.

5. EMP Simulators for Various Types of Nuclear EMPEnvironments an InterimCategorization, CARLEBAUM, member, IEEE. TRANSACTIONS ON ANTENNAS AND PROPAGATION, VOL. AP-26, NO. 1, JANUARY 1978. 